\title{
C/EBP $\beta$ promotes poly(ADP-ribose) polymerase inhibitor resistance by enhancing homologous recombination repair in high-grade serous ovarian cancer
}

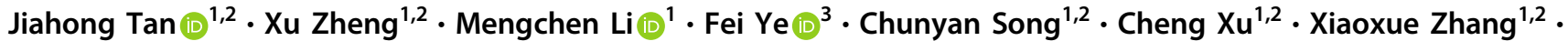 \\ Wenqian $\mathrm{Li}^{1,2} \cdot$ Ya Wang $\mathbb{1}^{1,2} \cdot$ Shaoqing Zeng $\mathbb{1}^{1,2} \cdot$ Huayi $\mathrm{Li} \mathbb{1}^{1,2} \cdot$ Gang Chen ${ }^{1,2} \cdot$ Xiaoyuan Huang $\mathbb{1}^{1,2}$. \\ Ding $\mathrm{Ma}^{1,2} \cdot$ Dan Liu $\mathbb{1}^{1,2} \cdot$ Qinglei Gao $\mathbb{1}^{1,2}$
}

Received: 10 September 2020 / Revised: 12 March 2021 / Accepted: 12 April 2021 / Published online: 8 May 2021

(c) The Author(s) 2021. This article is published with open access

\begin{abstract}
PARP inhibitors (PARPi) are efficacious in treating high-grade serous ovarian cancer (HG-SOC) with homologous recombination (HR) deficiency. However, they exhibit suboptimal efficiency in HR-proficient cancers. Here, we found that the expression of CCAAT/enhancer-binding protein $\beta(\mathrm{C} / \mathrm{EBP} \beta)$, a transcription factor, was inversely correlated with PARPi sensitivity in vitro and in vivo, both in HR-proficient condition. High C/EBP $\beta$ expression enhanced PARPi tolerance; PARPi treatment in turn induced $\mathrm{C} / \mathrm{EBP} \beta$ expression. $\mathrm{C} / \mathrm{EBP} \beta$ directly targeted and upregulated multiple HR genes (BRCA1, BRIP1, BRIT1, and RAD51), thereby inducing restoration of HR capacity and mediating acquired PARPi resistance. C/ EBP $\beta$ is a key regulator of the HR pathway and an indicator of PARPi responsiveness. Targeting C/EBP $\beta$ could induce HR deficiency and rescue PARPi sensitivity accordingly. Our findings indicate that HR-proficient patients may benefit from PARPi via targeting $\mathrm{C} / \mathrm{EBP} \beta$, and $\mathrm{C} / \mathrm{EBP} \beta$ expression levels enable predicting and tracking PARPi responsiveness during treatment.
\end{abstract}

These authors contributed equally: J. Tan, X. Zheng

Supplementary information The online version contains supplementary material available at https://doi.org/10.1038/s41388021-01788-4.
Dan Liu
tj_liudan@tjh.tjmu.edu.cn
$\triangle$ Qinglei Gao
qingleigao@hotmail.com

1 Cancer Biology Research Center (Key Laboratory of the Ministry of Education), Tongji Hospital, Tongji Medical College, Huazhong University of Science and Technology, Wuhan, People's Republic of China

2 Department of Obstetrics and Gynecology, Tongji Hospital, Tongji Medical College, Huazhong University of Science and Technology, Wuhan, People's Republic of China

3 Department of Neurosurgery, Tongji Hospital, Tongji Medical College, Huazhong University of Science and Technology, Wuhan, People's Republic of China

\section{Introduction}

Epithelial ovarian cancer is the most lethal gynecological cancer, accounting for more than $95 \%$ of ovarian malignancies [1]. Annually, there are 230000 new cases and 150 000 deaths worldwide [2]. High-grade serous ovarian cancer (HG-SOC) accounts for $75 \%$ of epithelial ovarian cancer, making it the most common histological type [2]. HGSOC is characterized by genomic instability, with acquired or inherited mutations in different DNA repair pathways [1].

To maintain genomic stability, organisms have evolved DNA damage responses (DDRs) to cope with constant endogenous and environmental genotoxic insults [3]. Double-strand breaks (DSBs) are extremely cytotoxic and difficult to repair [4]. The homologous recombination (HR) pathway is preferentially adopted to repair DSBs [5]. HRdeficient cells cannot utilize HR to repair DSBs, rendering them vulnerable to poly(ADP-ribose) polymerase inhibitors (PARPi) [6, 7]. Genomic analyses have revealed that HR deficiency (HRD) contributes to $~ 50 \%$ of HG-SOC cases $[6,8]$. Exposing these cells to PARPi will lead to DNA damage accumulation, cytotoxic genomic instability, and eventually synthetic lethality [9]. PARPi have been 
successfully implemented for recurrent HG-SOCs by leveraging inherent HRD [2].

Clinical trials have shown that patients with stable disease or partial response after platinum-based chemotherapy benefited from PARPi [1]. To date, olaparib, niraparib, and rucaparib have been approved for clinical application in platinum-sensitive recurrent HG-SOC [10]. PARPi show a range of efficacy on a continuum from patients with BRCA mutation, to those having HRD and even those without HRD [1, 10]. HR-deficient tumors, which have a "BRCAness profile", show increased response to platinum and PARPi [1, 6]. PARPi show suboptimal efficiency toward HR-proficient tumors, which constitute a non-negligible proportion of all HG-SOCs, owing to the insufficiency in inducing synthetic lethality $[6,8,11,12]$. Although PARPi activity extends beyond HRD, the exact distinctions between PARPi responders and non-responders remain unclear [6]. Currently, platinum sensitivity has been recognized as a functional indicator for PARPi application [13]. Tailoring precision medicine to individuals requires refining predictive biomarkers to help select patients who will benefit from PARPi [14].

Another issue related to PARPi application is the gradually emerging resistance $[13,14]$. Despite the efficacy of PARPi, many patients inevitably develop acquired resistance because of factors such as restoration of the HR function, secondary mutations, drug efflux, and new mutations in other DDR genes [13, 14]. Approaches to eliminate PARPi resistance warrant further investigation.

We have previously found that CCAAT/enhancer-binding protein $\beta(\mathrm{C} / \mathrm{EBP} \beta)$ is an important determinant of BRCA1-related DDR [15]. C/EBP $\beta$ is a basic leucine zipper transcription factor and implicated in many cellular biological processes, such as cell proliferation, differentiation, apoptosis, oncogene-induced senescence, and tumorigenesis [16]. Knocking down C/EBP $\beta$ suppresses the expression of several genes in BRCA1-related DDR pathways [15]. Moreover, C/EBP $\beta$ enhances platinum resistance and helps predict platinum sensitivity [15]. Besides, C/EBP $\beta$ is an independent prognostic factor for HG-SOC [15]. Therefore, we speculated that $\mathrm{C} / \mathrm{EBP} \beta$ may promote PARPi resistance.

In this study, we have investigated the relationship between $\mathrm{C} / \mathrm{EBP} \beta$ expression and PARPi responsiveness. We found that C/EBP $\beta$ promotes PARPi resistance by directly targeting and upregulating multiple key genes in the HR pathway. Furthermore, C/EBP $\beta$ could be exploited as an indicator of PARPi responsiveness and a therapeutic target. Targeting C/EBP $\beta$ will rescue PARPi sensitivity and provide a therapeutic target for HR-proficient tumors, thereby maximizing the effectiveness of PARPi in treating ovarian cancer.

\section{Results}

\section{C/EBP $\beta$ regulates DNA damage repair signals}

We had previously reported a significantly increased C/ EBP $\beta$ expression in HG-SOC [15]. To further clarify the role of $\mathrm{C} / \mathrm{EBP} \beta$ in ovarian cancer, TCGA RNAseqv2 dataset was analyzed. Among all the tumors surveyed, ovarian cancer showed the highest $\mathrm{C} / \mathrm{EBP} \beta$ expression (Fig. 1a). RNA-sequencing of the HR-proficient cell line $\mathrm{C} 13^{*}$ revealed that knocking down $\mathrm{C} / \mathrm{EBP} \beta$ changed its gene expression profile (Fig. 1b, Supplementary Table 1). Results of the RNA-sequencing were validated in two cancer cell lines (Supplementary Fig. S1a, S1b). Knockdown C/EBP $\beta$ significantly affected the KEGG pathway "Homologous recombination" (Fig. 1c, Supplementary Table 2). These differentially expressed genes (DEGs) also significantly accumulated in DDR-related Gene Ontologies (Fig. 1d; Supplementary Table 3). Therefore, we retrieved all pathways under "Replication and Repair" in the KEGG database, including ko03030 DNA replication, ko03410 Base excision repair, ko03420 Nucleotide excision repair, ko03430 Mismatch repair, ko03440 Homologous recombination, ko03450 Non-homologous end-joining, and ko03460 Fanconi anemia pathway, and obtained a total of 210 non-redundant genes. There was a significant overlap between the DEGs after C/EBP $\beta$ knockdown and these retrieved DDR genes $(P<0.0001$; Supplementary Fig. S1c). Depletion of C/EBP $\beta$ altered the expression profile of these 97 overlapping genes (Supplementary Fig. S1d). Consistent with enrichment analyses, the expression levels of four DDR genes, namely BRCA1, BRIP1, BRIT1, and RAD51, significantly correlated with $\mathrm{C} / \mathrm{EBP} \beta$ expression in the TCGA dataset, although some of them had no statistically significant expression difference (Supplementary Fig. S2). These four DDR genes were also downregulated after C/ EBP $\beta$ depletion in $\mathrm{C}_{13}{ }^{*}$ (Fig. 1e) and SKOV3 (Fig. 1f). These findings indicated that $\mathrm{C} / \mathrm{EBP} \beta$ regulated DDR signals in ovarian cancer.

\section{High C/EBP $\beta$ expression promotes PARPi resistance}

Accordingly, C/EBP $\beta$ might affect PARPi responsiveness. By exploring DepMap, we found that olaparib resistance of HR-proficient cancer cell lines positively correlated with C/ EBP $\beta$ expression (Supplementary Fig. S3). Endogenous C/ EBP $\beta$ levels were significantly correlated with olaparib resistance in vitro (Fig. 2a, b; Supplementary Fig. S4a). Cells having low C/EBP $\beta$ expression, such as OV2008 and A2780, showed low viability even at a small dose of olaparib, while $\mathrm{C} 13^{*}$, which exhibited strong C/EBP $\beta$ expression, survived under an increased olaparib concentration. Similar results were obtained using another two PARPi 

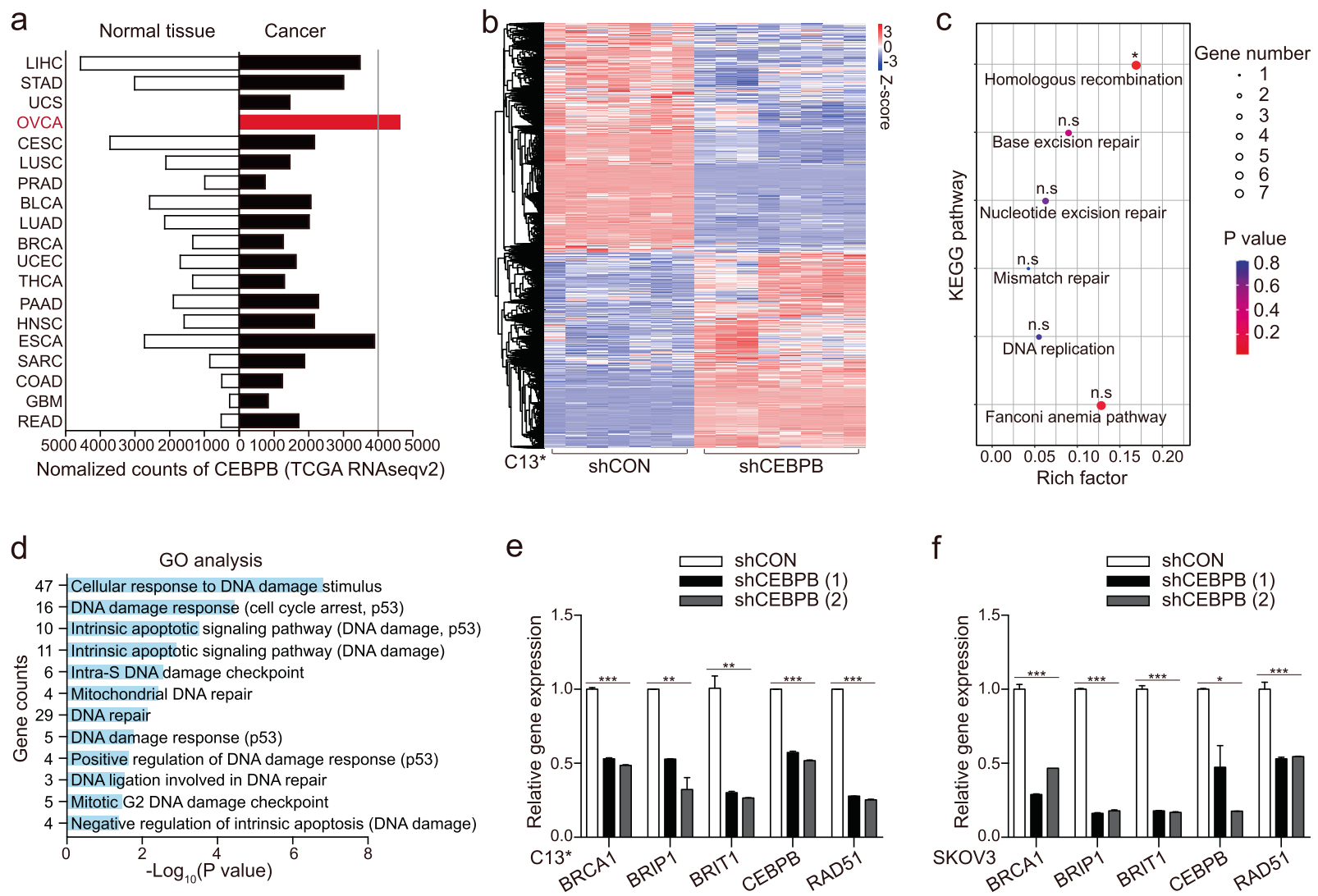

Fig. 1 C/EBPß regulates DNA damage repair signals. a TCGA RNAseqv2 data depicting CEBPB expression across a range of cancers. LIHC liver hepatocellular carcinoma, STAD stomach adenocarcinoma, UCS uterine carcinosarcoma, OVCA ovarian cancer, CESC cervical squamous cell carcinoma, LUSC lung squamous cell carcinoma, PRAD prostate adenocarcinoma, BLCA bladder urothelial carcinoma, LUAD lung adenocarcinoma, BRCA breast cancer, UCEC uterine corpus endometrial carcinoma, THCA thyroid carcinoma, PAAD pancreatic adenocarcinoma, HNSC head and neck squamous cell carcinoma, ESCA esophageal carcinoma, SARC sarcoma; COAD colon adenocarcinoma, GBM glioblastoma multiforme, READ rectum adenocarcinoma. RNA-sequencing of $\mathrm{C}_{13}{ }^{*} \operatorname{shCON}(N=7)$ and $\mathrm{C} 13^{*}$

AZD2461 and BMN673 (Supplementary Fig. S4b, c). Compared with OV2008 and A2780, C13* exhibited increased cell viability when treated with AZD2461 and BMN673. Under olaparib exposure, A2780 formed significantly lesser and smaller colonies, whereas the clonogenicity of $\mathrm{C}_{13}{ }^{*}$ was only slightly affected (Fig. 2c).

To further confirm these observations, we constructed $\mathrm{C}$ / EBP $\beta$ knock-in and knock-out cell lines using OV2008 and C13* (Fig. 2d) [17]. C/EBP $\beta$ knockdown sensitized C13* to olaparib, and C/EBP $\beta$ overexpression enhanced cell viability under olaparib exposure (Fig. 2e, Supplementary Fig. S4d). Similar results were observed in colony formation assays (Supplementary Fig. S4e, f). The effectiveness of olaparib treatment was verified using western blotting analysis of PAR (Supplementary Fig. S4g). The expression of $\mathrm{C} / \mathrm{EBP} \beta$ and cleaved caspase 3 varied in the opposite
shCEBPB $(N=8)$ were performed. b Heatmap of differentially expressed genes (DEGs) after C/EBP $\beta$ knockdown in $\mathrm{C} 13^{*}$. DEGs were subjected to enrichment analysis. c The effects of $\mathrm{C} / \mathrm{EBP} \beta$ knockdown on DDR-related KEGG pathways. d Enriched Gene Ontologies after C/EBP $\beta$ knockdown. The expression of the four HR genes, whose expression was significantly correlated with $\mathrm{C} / \mathrm{EBP} \beta$ expression, was detected in (e) $\mathrm{C}_{13}{ }^{*}$ and (f) SKOV3 after C/EBP $\beta$ manipulation. Each sample had triplicates and data were presented as mean \pm SEM of three experiments (Student's $t$ test). $P$ value was denoted as $* P<0.05$, $* * P<0.01$, and $* * * P<0.001$, "n.s" represents "not significant".

direction further supported that C/EBP $\beta$ promoted PARPi resistance (Supplementary Fig. S4h). Stably transfected C13* or OV2008 cells were subcutaneously inoculated into mouse flanks. At the indicated time, tumor sizes were monitored using in vivo bioluminescent imaging. High $\mathrm{C} /$ EBP $\beta$ expression conferred olaparib tolerance in vivo. Tumor growth was only slightly suppressed by olaparib in mice engrafted with $\mathrm{C} 13^{*}$ shCON cells, while mice inoculated with $\mathrm{C} 13^{*}$ shCEBPB cells exhibited decreased luciferase expression and were sensitive to olaparib (Fig. 2f). Xenografts models of OV2008 CON cells showed sensitivity toward olaparib, wherein tumors were restrained and even eliminated, while the C/EBP $\beta$-overexpressing group was insensitive to olaparib and had heavier tumor burdens (Supplementary Fig. S4i). We also examined the effect of $\mathrm{C} / \mathrm{EBP} \beta$ on survival, with survival cut-off criteria defined as 

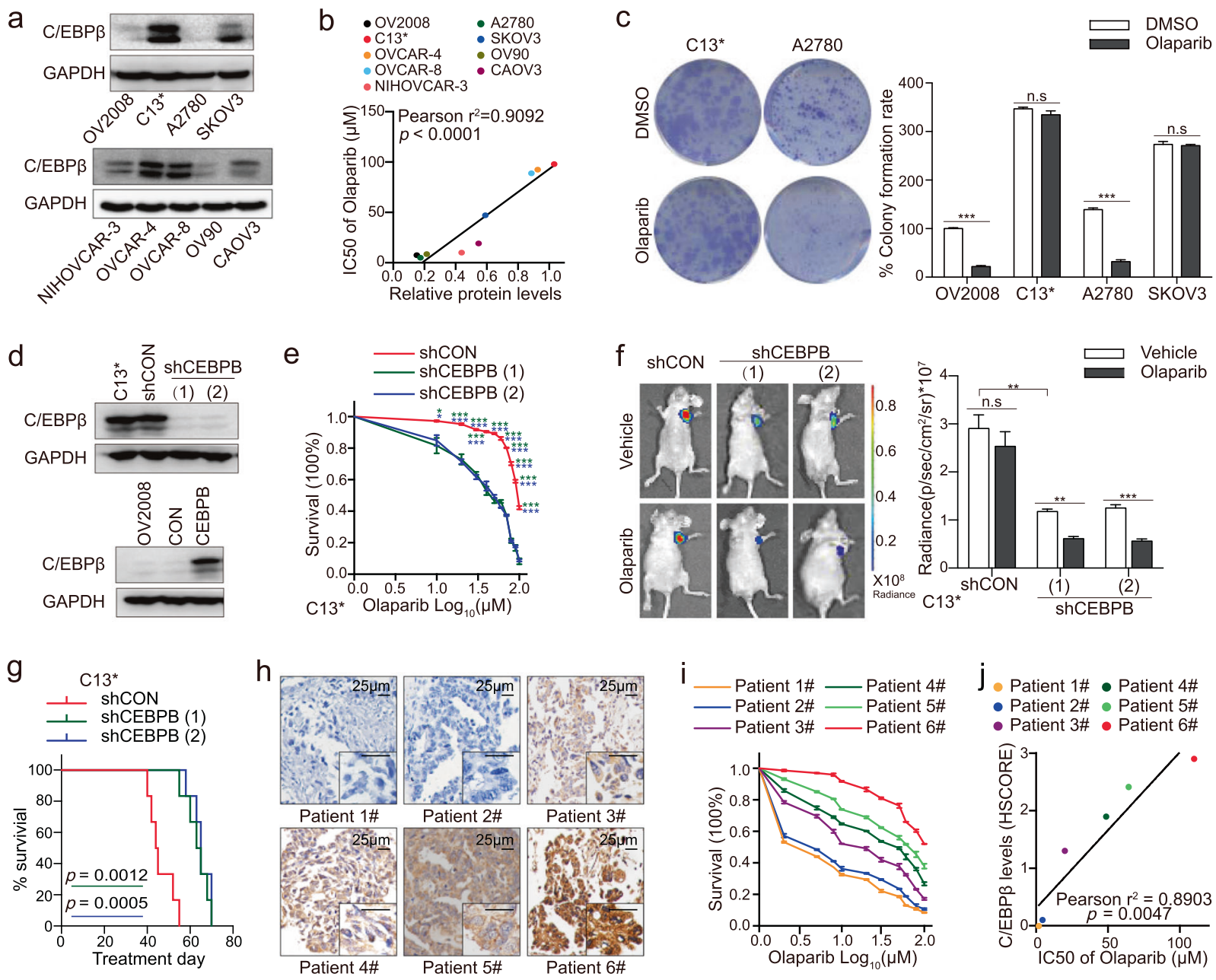

Fig. 2 High C/EBPß expression promotes PARPi resistance. a Western blotting analysis detected C/EBP $\beta$ expression in cancer cell lines. The experiment was repeated thrice and representative images were shown. b Correlation analysis of C/EBP $\beta$ expression levels and IC50s of cancer cell lines for olaparib (Pearson's correlation test). c Colony-formation rates of cancer cell lines under olaparib exposure. Each assay was conducted in triplicates and error bars indicated mean \pm SEM of three assays (Student's $t$ test). Lentiviruses were used to modulate $\mathrm{C} / \mathrm{EBP} \beta$ expression. $\mathbf{d}$ After transfection, $\mathrm{C} / \mathrm{EBP} \beta$ expression was detected using western blot. A representative blot of three assays was shown. e Cell viability assays for C/EBP $\beta$-depleted $\mathrm{C} 13^{*}$ cells (Student's $t$ test). Each sample had six replicates and all experiments had triplicates. Error bars represented mean \pm SEM. Stably transfected C13* cells were subcutaneously inoculated into mouse flanks. Olaparib

tumor volume reaching $1500 \mathrm{~mm}^{3}$ or an observation duration of 90 days. C/EBP $\beta$ depletion substantially improved survival compared with that of the control (Fig. $2 \mathrm{~g}$ ), while $\mathrm{C} / \mathrm{EBP} \beta$ overexpression deteriorated survival outcome (Supplementary Fig. S4j). C/EBP $\beta$ expression was also interfered with siRNA in OV2008 and A2780 cells. Depletion of $\mathrm{C} / \mathrm{EBP} \beta$ did not further enhance PARPi responsiveness (Supplementary Fig. S5a-S5d).

The relationship between C/EBP $\beta$ expression and olaparib sensitivity was also examined in six primary cultures. or vehicle was administered (six mice per group). f Tumor sizes were monitored using in vivo bioluminescent imaging, and tumor burdens were quantified by total radiance. Representative images of mouse xenografts were shown (Student's $t$ test). Error bar, mean \pm SEM. g Kaplan-Meier survival plot of mice after C/EBP $\beta$ manipulation and olaparib treatment (Log-rank test). Freshly collected specimens were used for primary cultures. $\mathbf{h}$ Immunohistochemical analysis of C/EBP $\beta$ expression in tumor tissues. i Cell viability assays of primary cultures. Each assay was conducted in six wells and repeated three times. Error bars depicted mean \pm SEM. $\mathbf{j}$ Correlation analysis of C/EBP $\beta$ expression levels and IC50s of primary cultures for olaparib (Pearson's correlation test). $P$ value was denoted as $* P<0.05$, $* * P<0.01$, and *** $P<0.001$, "n.s" represents "not significant".

After confirmation of epithelial content (Supplementary Fig. S5e), cell viability assays were performed. Cell survival upon olaparib treatment was directly proportional to $\mathrm{C} /$ EBP $\beta$ expression (Fig. 2h, i; Supplementary Fig. S5f). Correlation analysis showed that olaparib resistance was positively related to C/EBP $\beta$ expression $\left(r^{2}=0.8903, P=\right.$ 0.0047; Fig. 2j). The negative relation between C/EBP $\beta$ and cleaved caspase 3 was also observed (Supplementary Fig. $\mathrm{S} 5 \mathrm{~g}, \mathrm{~h}$ ), but the relation between $\mathrm{C} / \mathrm{EBP} \beta$ and $\mathrm{Ki}-67$ in the six patients was not significant (Supplementary Fig. S5i). 
To explore the influences of C/EBP $\beta$ on apoptosis, expression of cleaved caspase 8 and cleaved PARP1 was also examined (Supplementary Fig. S6a, b). Depletion of C/ EBP $\beta$ exacerbated apoptosis in $\mathrm{C} 13^{*}$ cells, which could be rescued by adding the caspase inhibitor Q-VD-OPh, while overexpressed C/EBP $\beta$ attenuated apoptosis in OV2008 cells (Supplementary Fig. S6c, d). C/EBP $\beta$ also affected ROS levels in $\mathrm{C}_{13}{ }^{*}$ and OV2008 cells and regulated the expression of reductases GSTP1 and NQO1 (Supplementary Fig. S7). Taken together, C/EBP $\beta$ expression was inversely correlated with PARPi sensitivity.

\section{Olaparib treatment induces C/EBP $\beta$ expression}

To clarify the reciprocal relationship between C/EBP $\beta$ and PARPi responsiveness, we treated A2780 and OV2008 cells with olaparib at different concentrations and for different periods. Surprisingly, Olaparib induced C/EBP $\beta$ expression in a dose-dependent manner and also in a time-dependent manner (Fig. 3a, b; Supplementary Fig. S8a, b).

A stable resistant cell strain was generated by culturing A2780 in the continued presence of olaparib. Resistance emerged with a gradual increase in exposure. When the IC50 of the resistant strain was $\geq 30$ times that of the parent line, an olaparib-resistant strain had then been developed and referred to as A2780 OlaR. When exposed to the same concentration of olaparib, A2780 OlaR formed more colonies and had less apoptosis (Fig. 3c, Supplementary Fig. S8c). By cell survival assays, we obtained similar results (Fig. 3d). Consistently, A2780 OlaR expressed less cleaved capsase3 than A2780 (Supplementary Fig. S8d). We had also ceased olaparib treatment for one month, which had a a

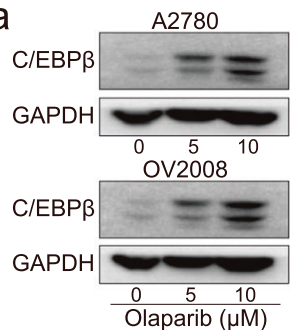

d

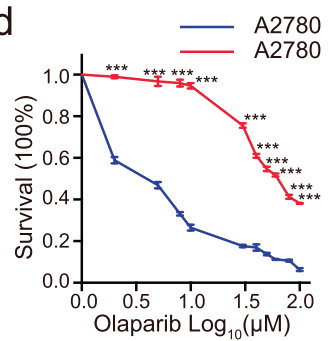

h

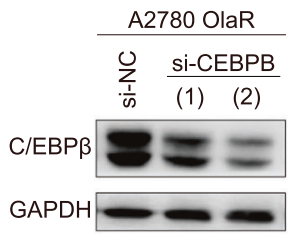

b

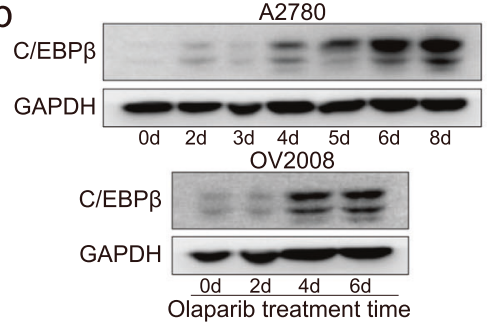

e

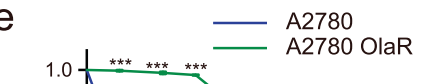

C

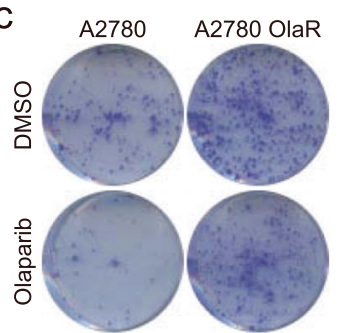

f

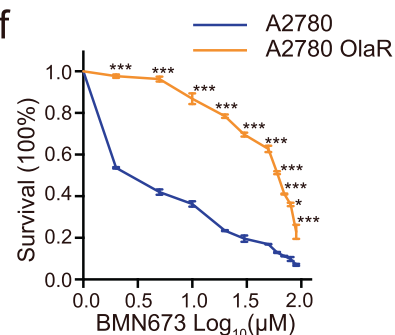

j

i

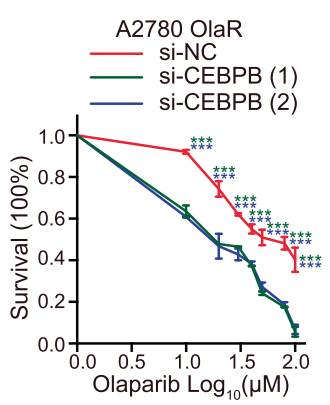

Fig. 3 Olaparib treatment induces C/EBP $\beta$ expression. A2780 and OV2008 were exposed to olaparib for (a) different concentrations and (b) different periods. Subsequently, C/EBP $\beta$ expression was detected by western blot and the experiments were repeated three times. A2780 was exposed to olaparib to generate the resistant strain A2780 OlaR. c Colony-formation rates of A2780 and A2780 OlaR under olaparib exposure. Each sample had triplicate wells and data represented mean \pm SEM of three assays (Student's $t$ test). Cell viability assays for A2780 and A2780 OlaR under different concentrations of (d) olaparib, (e) AZD2461, and (f) BMN673. Each sample had six replicates and error bars were mean \pm SEM of three experiments (Student's $t$ test). $\mathbf{g}$

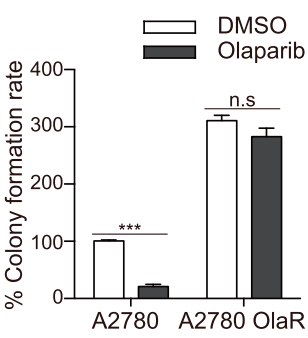

g
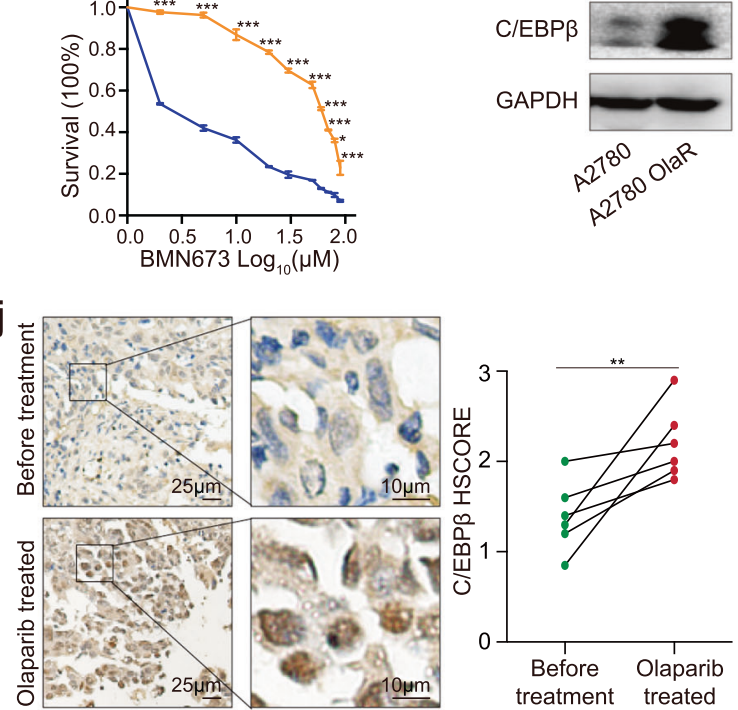

$\mathrm{C} / \mathrm{EBP} \beta$ expression in A2780 OlaR was detected by western blot and repeated three times. $\mathbf{h}$ siRNA interference of $\mathrm{C} / \mathrm{EBP} \beta$ expression was used in A2780 OlaR. Western blotting analysis was performed and repeated thrice. i Cell viability assays for A2780 OlaR after CEBPB interference. The experiment was repeated for thrice in six replicates for each cell line and error bars depicted mean \pm SEM (Student's $t$ test). $\mathbf{j}$ Immunohistochemical staining of C/EBP $\beta$ in paired specimens before and after olaparib treatment (Student's $t$ test). $P$ value was denoted as $* P<0.05, * * P<0.01$, and $* * * P<0.001$, "n.s" represents "not significant". 
hardly measurable influence on the drug tolerance of A2780 OlaR (Supplementary Fig. S8e). Cell cycle analysis showed subtle changes between A2780 and A2780 OlaR, implying that the alteration of drug responsiveness was independent of the cell cycle (Supplementary Fig. S8f). Concurrently, resistance was maintained when A2780 OlaR was treated with AZD2461 or BMN673 (Fig. 3e, f).

We then examined C/EBP $\beta$ expression in A2780 OlaR and observed a pronounced increase in $\mathrm{C} / \mathrm{EBP} \beta$ expression (Fig. 3g, Supplementary Fig. S8g). C/EBP $\beta$ elimination by siRNA interference rendered A2780 OlaR sensitive to olaparib without affecting cell viability (Fig. 3h, i; Supplementary Fig. S8h).

Clinical specimens obtained from patients who had HRproficient HG-SOC and had undergone several rounds of olaparib treatment were used to further explore the mutual relationship between $\mathrm{C} / \mathrm{EBP} \beta$ and PARPi. We found increased C/EBP $\beta$ expression after olaparib treatment (Fig. $3 \mathrm{j}$ ), along with decreased cleaved caspase 3 expression (Supplementary Fig. S9). The effect of C/EBP $\beta$ on PARPi responsiveness was also observed in the HR-proficient breast cancer cell line MDA-MB-231, but not in the BRCA1-mutant MDA-MB-436 (Supplementary Figs. S10, S11). In summary, PARPi administration induced C/EBP $\beta$ expression, and C/EBP $\beta$ in turn promoted PARPi resistance.

\section{C/EBP $\beta$ directly targets HR genes and affects DNA damage repair}

To determine the mechanisms underlying the effects of $\mathrm{C} /$ EBP $\beta$ on PARPi resistance, we performed RNA-sequencing of A2780 OlaR and its parent line A2780 (Supplementary Fig. S12a, b; Supplementary Table 4). Surprisingly, these DEGs significantly overlapped with the DEGs regulated by C/EBP $\beta$ ( $p<0.0001$; Fig. 4a). These results implied that $\mathrm{C} /$ EBP $\beta$ was strongly correlated with the transformation from sensitivity to resistance.

Since $C / E B P \beta$ is a transcription factor, we checked the functional categories of C/EBP $\beta$ targets by reanalyzing the chromatin immunoprecipitation sequencing (ChIP-seq) data [15]. We found that $20 \%$ of HR genes were C/EBP $\beta$ targets (Supplementary Fig. S12c). The binding affinity of C/EBP $\beta$ for the HR genes was then predicted in Cistrome DB. Four HR genes, namely BRCA1, BRIP1, BRIT1, and RAD51, were found to be potential targets of C/EBP $\beta$ (Fig. 4b). All four genes scored high at both $\mathrm{C} / \mathrm{EBP} \beta$ motifs when validated in JASPAR (Supplementary Fig. S12d, Supplementary Table 5). This finding was further confirmed by ChIPqPCR, where the high binding affinity of C/EBP $\beta$ for these four genes was significantly abolished after $\mathrm{C} / \mathrm{EBP} \beta$ knockdown (Fig. 4c). Promoter luciferase reporter plasmids for these four HR genes were constructed. Depletion of
C/EBP $\beta$ significantly repressed the luciferase activity of reporter plasmids (Fig. 4d). These results indicated that C/ EBP $\beta$ directly targeted these four HR genes.

To ascertain the effects of C/EBP $\beta$ on HR-mediated repair of DNA damage, HR reporter assays were performed. $\mathrm{C} / \mathrm{EBP} \beta$ knockdown reduced the ratio of $\mathrm{GFP}^{+}$cells, while overexpressed C/EBP $\beta$ enhanced HR efficiency as indicated by more $\mathrm{GFP}^{+}$cells (Fig. 4e; Supplementary Fig. S12e, S12f). To evaluate DNA damage, comet assay and $\gamma \mathrm{H} 2 \mathrm{AX}$ staining were performed [18]. Olaparib severely impaired DNA and induced more DNA in tails after C/EBP $\beta$ knockdown, while $\mathrm{C} / \mathrm{EBP} \beta$ overexpression helped maintain DNA integrity and diminished DNA in tails (Fig. 4f, Supplementary Fig. S13a). C/EBP $\beta$ depletion triggered $\gamma \mathrm{H} 2 \mathrm{AX}$ foci formation in $\mathrm{C}_{13}{ }^{*}$ cells and accumulated DNA damage, whereas exogenous augmentation of C/EBP $\beta$ in OV2008 cells inhibited $\gamma \mathrm{H} 2 \mathrm{AX}$ foci formation and protected cells from DNA damage (Supplementary Fig. S13b, c). Together, C/EBP $\beta$ directly targeted four HR genes and protected cells from DNA damage, thereby mediating PARPi resistance.

\section{C/EBP $\beta$ upregulates HR genes and induces HR restoration}

HR gene panel perturbations after C/EBP $\beta$ manipulation were assessed. C/EBP $\beta$ knock-in upregulated HR genes expression in OV2008, while C/EBP $\beta$ knock-out reduced their expression in $\mathrm{C}_{13}{ }^{*}$ (Fig. 5a, Supplementary Fig. S14a, b). In A2780 OlaR, C/EBP $\beta$-targeted siRNA interference decreased the expression of all four genes (Fig. 5b, Supplementary Fig. S14c). Similar results were noted in cancer cell lines, where HR genes expression varied along with $\mathrm{C} /$ EBP $\beta$ expression (Fig. 5c). Formation of HR proteins foci serves as an indicator of HR capacity [19]. Under olaparib exposure, C/EBP $\beta$ depletion suppressed HR proteins foci formation in $\mathrm{C}_{13}{ }^{*}$ (Fig. 5d), while C/EBP $\beta$ overexpression elevated their intranuclear foci formation rates in OV2008 (Supplementary Fig. S14d).

The effects of C/EBP $\beta$ on the HR pathway were maintained in vivo. $\mathrm{C}_{13}{ }^{*}$ and OV2008 mouse models were treated with $30 \mathrm{mg} / \mathrm{Kg}$ olaparib/vehicle intraperitoneally every 2 days for 40 days and the mice were sacrificed for tissue harvest after bioluminescent imaging. Immunohistochemical analyses of the four HR genes recapitulated our in vitro experiments. $\mathrm{C} / \mathrm{EBP} \beta$ depletion inhibited their expression, whereas their expression was enhanced by $\mathrm{C} /$ EBP $\beta$ overexpression (Fig. 5e; Supplementary Figs. S14e, f, and S15a). The same tendency was observed at their mRNA expression levels (Supplementary Fig. S15b, c). Similarly, cleaved caspase 3 changed opposite to $\mathrm{C} / \mathrm{EBP} \beta$, but no significant variation of $\mathrm{Ki}-67$ expression was observed (Supplementary Fig. S15d-g). Overall, C/EBP $\beta$ upregulated HR genes and induced recovery of HR capacity. 
a

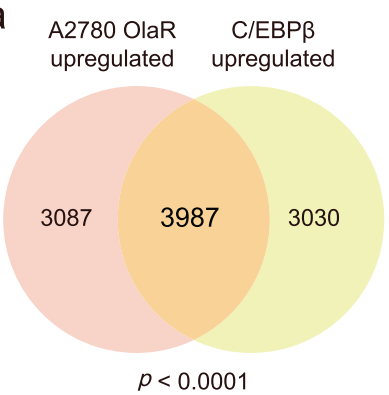

C

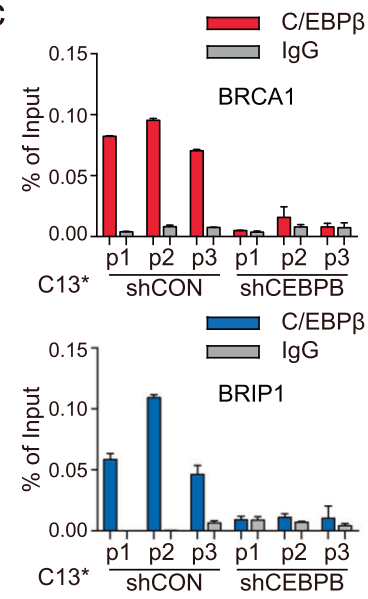

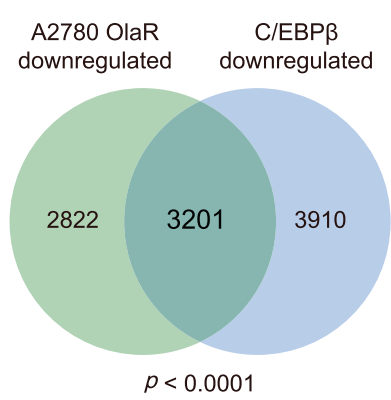

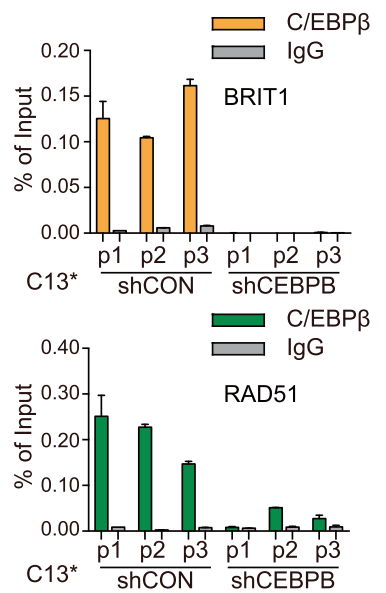

b

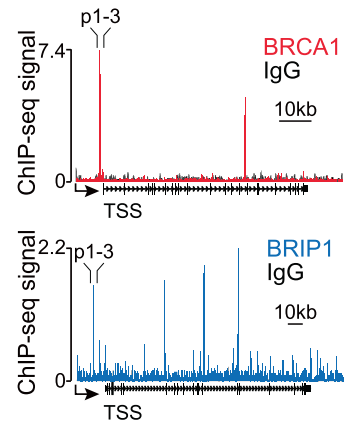

d
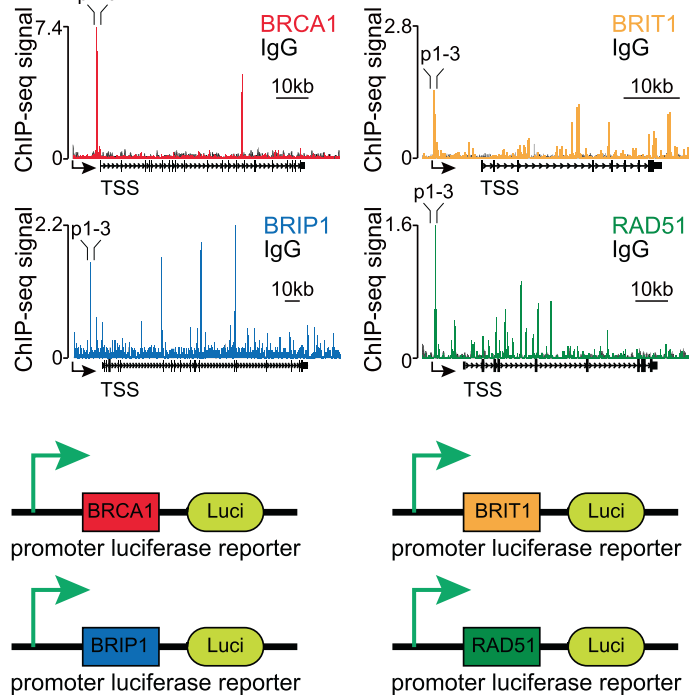

promoter luciferase reporter

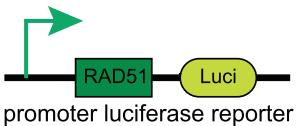

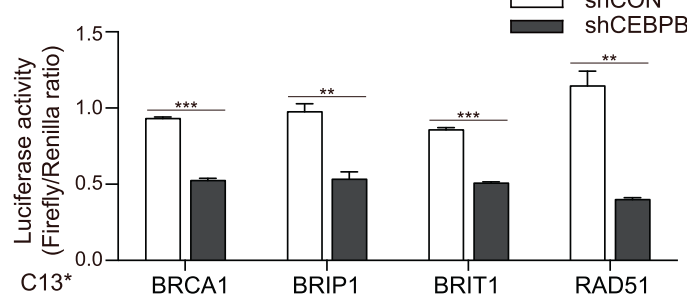

f
$\mathrm{C} 13^{*}$
shCON
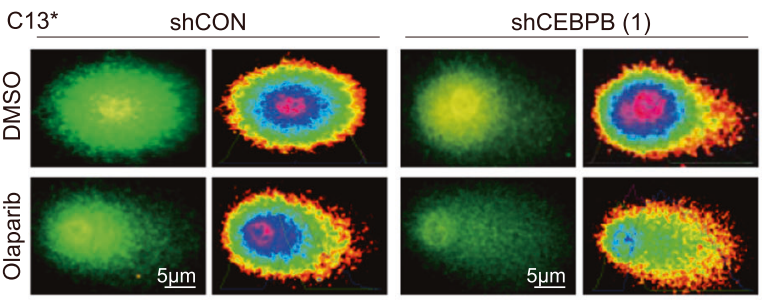

ShCEBPB (2)
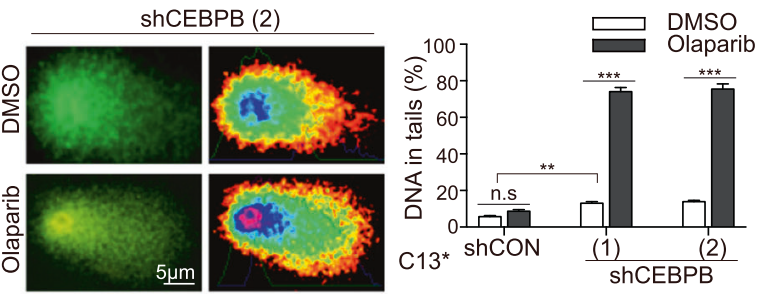

of the four HR genes in $\mathrm{C} 13^{*}$ shCON and shCEBPB cells. The experiments were conducted in triplicates and repeated three times. Data were presented as mean \pm SEM (Student's $t$ test). e HR reporter assays in $\mathrm{C} 13^{*}$ shCON and shCEBPB cells after olaparib treatment. Each sample had triplicates and error bars represented mean \pm SEM of three experiments (Student's $t$ test). $\mathbf{f}$ Comet assays with $\mathrm{C}_{13}{ }^{*}$ shCON and shCEBPB cells after olaparib treatment. Results were obtained from three independent experiments and depicted as mean \pm SEM (Student's $t$ test). $P$ value was denoted as $* P<0.05$, $* * P<0.01$, and $* * * P<0.001$, "n.s" represents "not significant". 
a

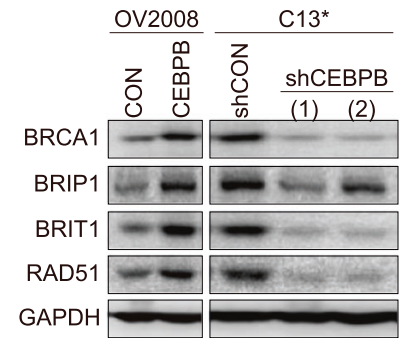

b

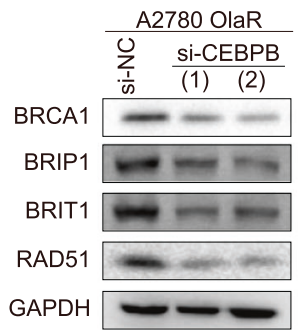

C

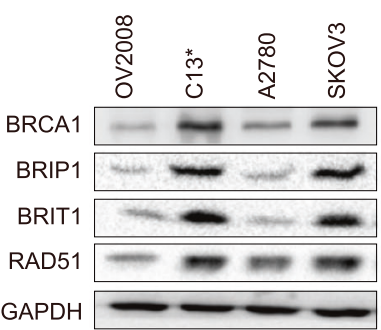

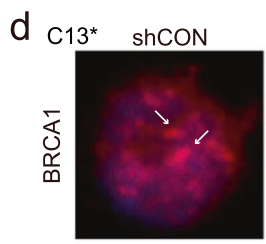

shCEBPB
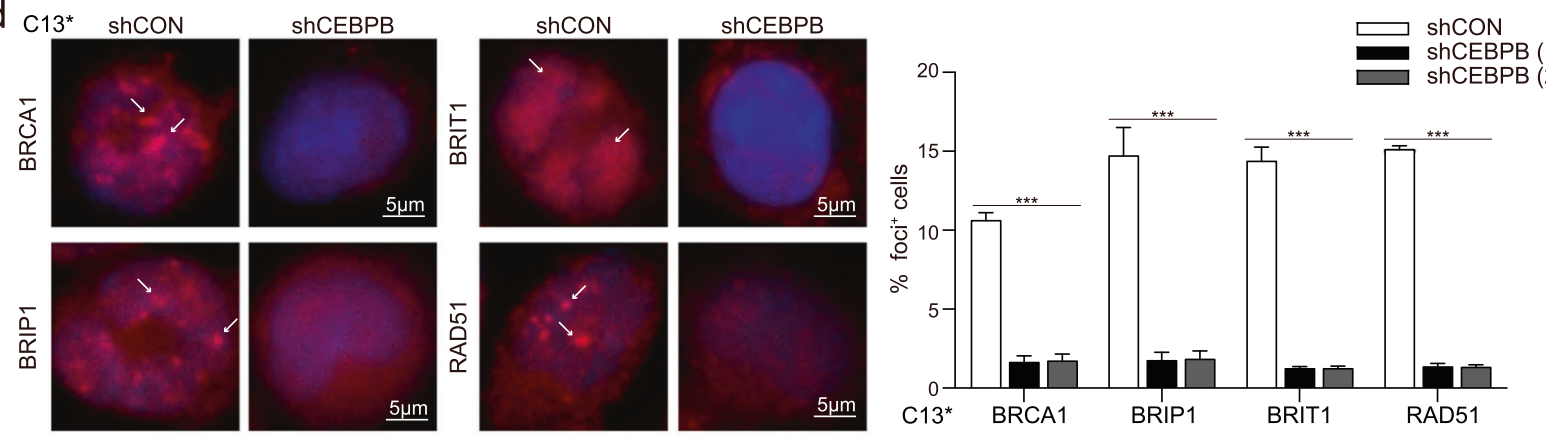

e
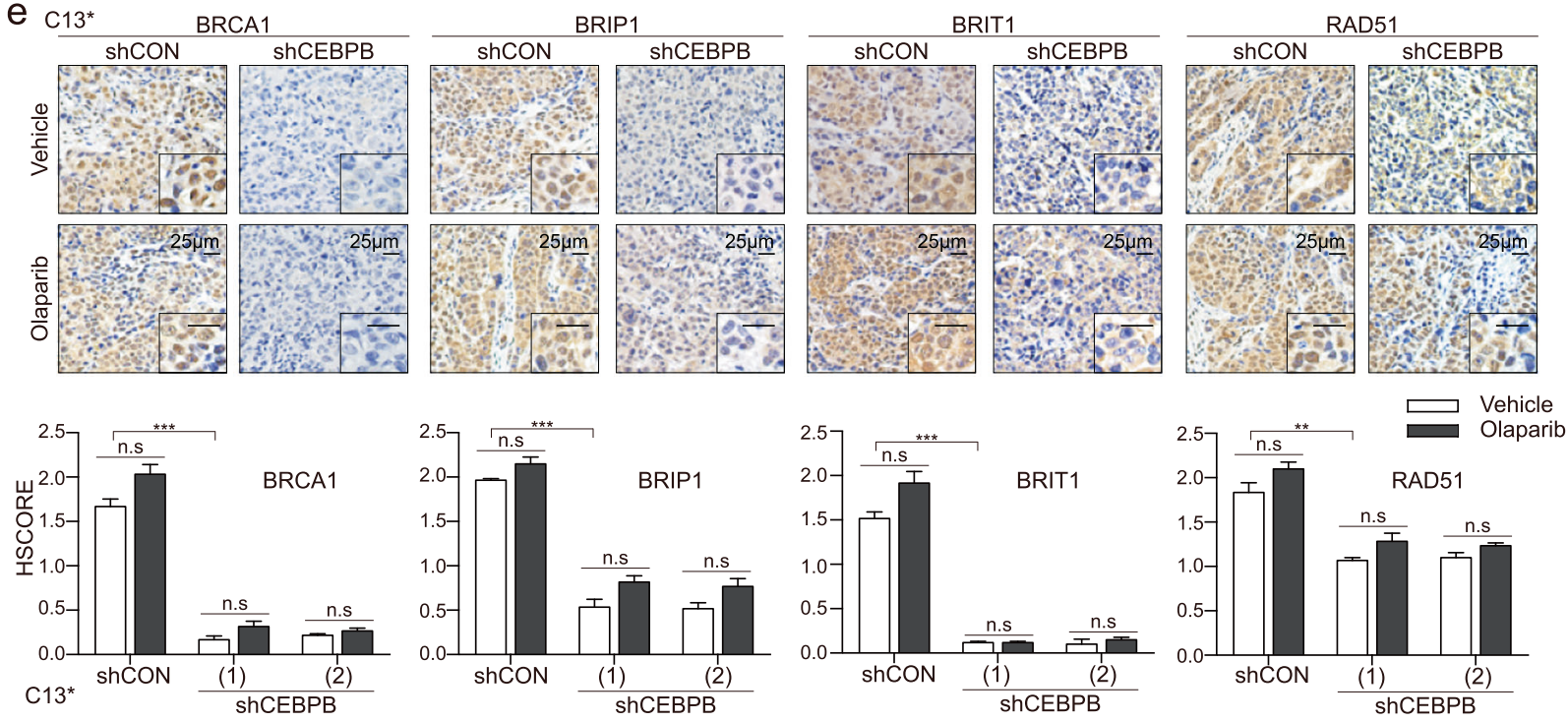

Fig. 5 C/EBP $\beta$ upregulates HR genes and induces HR restoration. a HR genes expression was detected by western blot after C/EBP $\beta$ manipulation in OV2008 and $\mathrm{C}_{1}{ }^{*}[35]$. b HR genes expression in A2780 OlaR was detected using western blot after C/EBP $\beta$ knockdown. $\mathbf{c}$ HR genes expression in the four mainly used cancer cell lines was detected by western blot. By western blotting analysis, all experiments were repeated three times. d Immunofluorescence staining of HR proteins; representatives were shown (left). Staining foci were indicated by arrowheads. HR protein foci formation rates in $\mathrm{C}_{13}{ }^{*}$

\section{C/EBP $\beta$ promotes PARPi resistance through the HR pathway}

To rescue PARPi sensitivity in C/EBP $\beta$-overexpressing cells, the expression of the four HR genes were depleted. siRNA interference of the four HR genes decreased the ratio of $\mathrm{GFP}^{+}$cells in OV2008 CEBPB cells, indicating reduced HR efficiency (Fig. 6a, b). Similar results were observed in

shCON and shCEBPB cells after olaparib treatment were calculated (right). Data were obtained from three independent experiments and denoted as mean \pm SEM (Student's $t$ test). e Tumor tissues from $\mathrm{C} 13^{*}$ mouse models (six mice per group) were stained with the indicated antibody, and HR proteins expression was evaluated using HSCORE. Data shown represented mean \pm SEM (Student's $t$ test). $P$ value was denoted as $* P<0.05, * * P<0.01$, and $* * * P<0.001$, "n.s" represents "not significant".

A2780 OlaR after siRNA interference (Supplementary Fig. S16a, b). Silencing of the four HR genes attenuated RAD51 foci formation rates in OV2008 CEBPB and A2780 OlaR (Supplementary Fig. S16c, d). $\gamma \mathrm{H} 2 \mathrm{AX}$ staining showed pronounced foci formation rate after siRNA transfection in OV2008 CEBPB cells (Fig. 6c). Consistent results were obtained in A2780 OlaR (Supplementary Fig. 17a). Cell viability assays showed that depletion of any of these four 


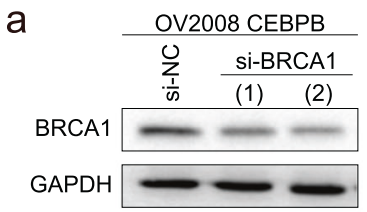

b

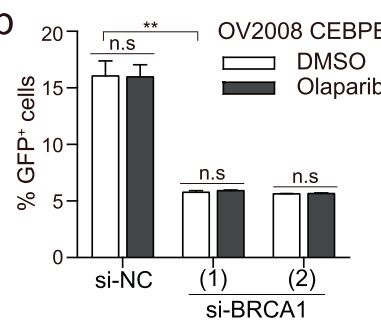

C

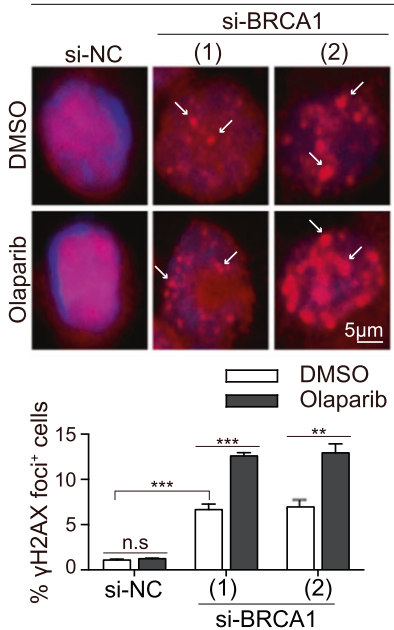

d

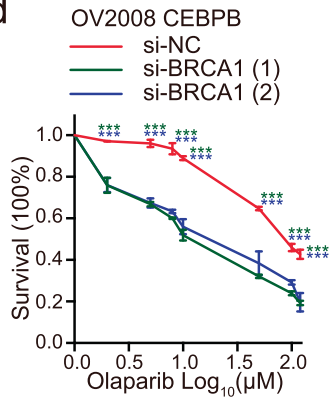

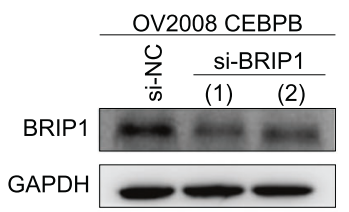

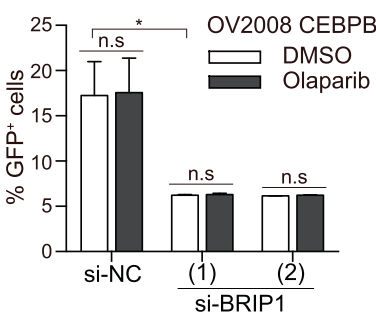

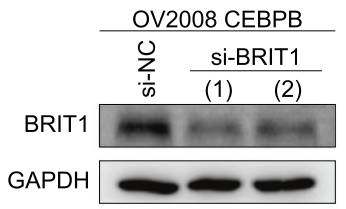
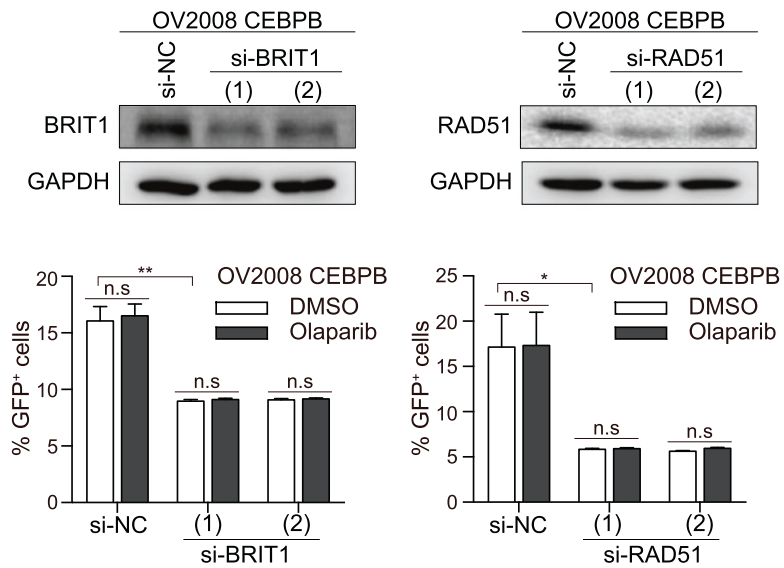

OV2008 CEBPB
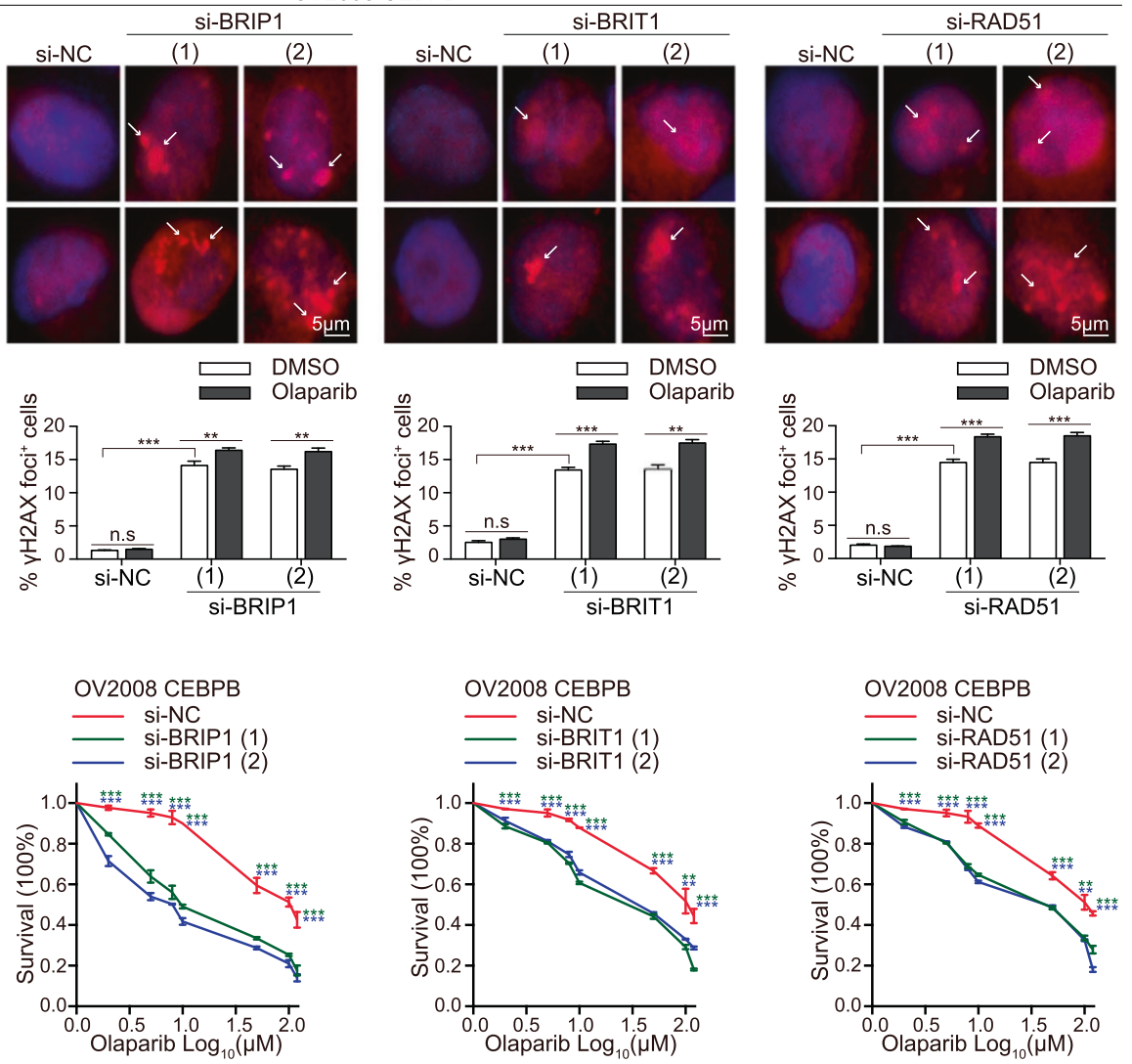

Fig. 6 siRNA interference of HR genes abolishes the effects of $\mathrm{C} /$ EBPß. C/EBP $\beta$-overexpressing OV2008 cells were transfected with siRNA targeting BRCA1, BRIP1, BRIT1, or RAD51. a HR protein expression was detected by western blot after siRNA interference and repeated thrice. b HR reporter assays were performed after siRNA interference and olaparib treatment. Each sample had triplicates and the data represented mean \pm SEM of three experiments (Student's $t$ test). c Immunofluorescence staining of $\gamma \mathrm{H} 2 \mathrm{AX}$; representatives were shown (top). Staining foci were indicated by arrowheads. $\gamma \mathrm{H} 2 \mathrm{AX}$ foci

HR genes would enhance olaparib sensitivity (Fig. 6d, Supplementary Fig. S17b). Collectively, the findings indicated that $\mathrm{C} / \mathrm{EBP} \beta$ promoted PARPi resistance through the HR pathway.
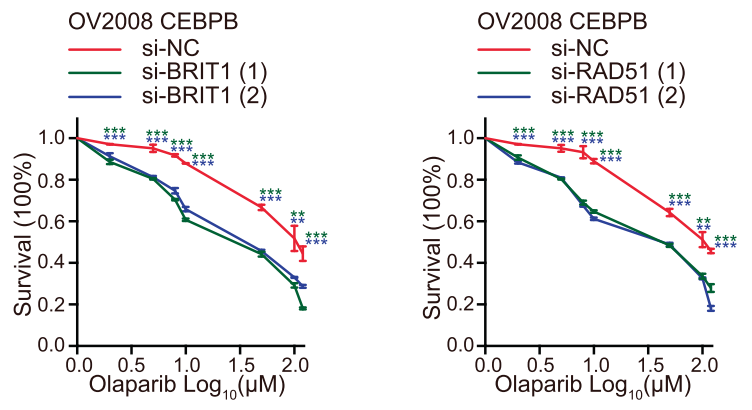

formation rates were calculated after confirmation of transfection and olaparib treatment (bottom). Results were obtained from three independent experiments and presented as mean \pm SEM (Student's $t$ test). d Cell viability assays under olaparib exposure after siRNA interference. The experiments were conducted thrice with six replicate wells. Data were shown as mean \pm SEM (Student's $t$ test). $P$ value was denoted as $* P<0.05$, $* * P<0.01$, and $* * * P<0.001$, "n.s" represents "not significant".

\section{Discussion}

Exploitation of dysregulated DDR is a highly promising approach to develop anti-cancer treatment [20]. C/EBP $\beta$, a 
basic leucine zipper transcription factor, was found to affect BRCA1-related DDR [15]. In this study, we explored the reciprocal relationship between $\mathrm{C} / \mathrm{EBP} \beta$ and PARPi responsiveness. The results herein showed that $\mathrm{C} / \mathrm{EBP} \beta$ affected DDR signals, especially the HR pathway of ovarian cancer. C/EBP $\beta$ directly targeted and upregulated multiple HR genes, including BRCA1, BRIP1, BRIT1, and RAD51, inducing restoration of HR capacity and promoting PARPi resistance. Meanwhile, olaparib treatment induced C/EBP $\beta$ expression, implying that $\mathrm{C} / \mathrm{EBP} \beta$ contributed to acquired PARPi resistance. Thus, $\mathrm{C} / \mathrm{EBP} \beta$ could be exploited as an indicator of PARPi responsiveness and as a therapeutic target. Targeting C/EBP $\beta$ could induce HRD and rescue PARPi sensitivity accordingly.

$\mathrm{HR}$, an error-free form of repair with high fidelity, is crucial for cell survival $[5,21]$. C/EBP $\beta$ directly targeted and upregulated four key HR genes. BRCA1 plays a role in transcription, DNA repair of DSBs, and recombination. BRIP1 interacts with BRCA1 and the bound complex is important in the normal repair function of BRCA1. BRIT1 is a DDR protein and engages in G2/M checkpoint arrest. RAD51 functions in homologous pairing and strand transfer of DNA. C/EBP $\beta$ knockdown decreased the expression of these genes and reduced HR capacity. The effects of $\mathrm{C} /$ EBP $\beta$ on HR genes may not be confined to transcription regulation, which might further reinforce its potential. Besides, some other important HR genes, like BABAM1, were downregulated by $\mathrm{C} / \mathrm{EBP} \beta$ and involved in the transition from PARPi sensitivity to resistance. Therefore, C/ EBP $\beta$ is potentially an important regulator of the HR pathway, providing a rationale for targeting C/EBP $\beta$ to induce HRD in HR-proficient tumors. Except for the HR pathway, the functional categories of C/EBP $\beta$ targets involve several DNA repair pathways [15]. To exclude the influences of mismatch repair (MMR), MMR-proficient cell lines $\left(\mathrm{C} 13^{*}, \mathrm{OV} 2008\right.$, and A2780) and MMR-deficient SKOV3 were used in this study [22-24]. Since there might be compensatory upregulation of other DNA repair pathways upon hypofunction of the HR pathway, C/EBP $\beta$ depletion cannot make the HR-proficient cancer cells as sensitive as the BRCA-mutated cells. However, the enhanced sensitivity deserves further exploitation.

PARPi, which specifically kill cancer cells with inadequate HR capacity, have benefited many HG-SOC patients $[2,10]$. The emergence of resistance has precluded their further application; 40-70\% of patients could not respond as expected to olaparib because of drug resistance [13, 14, 25]. In-depth research has been performed on specific predispositions to PARPi resistance [26-30]. Combination therapies were explored, but their uses were hindered by sequential orders and dose-limiting toxicity [31-35]. In this study, we used three PARPi: olaparib, AZD2461, and BMN673. Olaparib inhibits PARP1/2;
AZD2461 is a poor substrate of p-glycoprotein and inhibits PARP1/2/3; BMN673 suppresses PARP1/2/3/4 and has the strongest PARP trapping ability [36-39]. All three PARPi could not trigger sufficient efficiency in $\mathrm{C} /$ EBP $\beta$-overexpressing cells. Patients with endogenous overexpressed C/EBP $\beta$ were inherently resistant to PARPi. ROS was reported to have a role in PARPi responsiveness [40]. C/EBP $\beta$ could augment the expression of reductases to neutralize ROS levels and affect ROS distribution, which also contributed to olaparib tolerance. Besides, the effect of $\mathrm{C} / \mathrm{EBP} \beta$ was also observed in breast cancer cell line MDAMB-231. Although there was no overt effect of C/EBP $\beta$ in the BRCA1 mutant MDA-MB-436, which was sensitive to PARPi. These findings indicate that $C / E B P \beta$ is an important and general mediator of PARPi resistance. Given that both platinum- and PARPi-based therapies function through affecting DNA repair machinery, there is cross-resistance between platinum and PARPi $[2,10,41]$. Platinum-resistant HG-SOC is not susceptible to PARPi [1, 2]. C/EBP $\beta$ has been previously found to enhance platinum resistance; $\mathrm{C} 13^{*}$ and OV2008 represents cisplatin-resistant and cisplatinsensitive cell line pair [15]. C/EBP $\beta$ knockdown could promote olaparib sensitivity in $\mathrm{C} 13^{*}$. Therefore, targeting $\mathrm{C} / \mathrm{EBP} \beta$ also helps overcome this cross-resistance.

Predictive biomarkers for PARPi response are urgently required [14]. Although platinum sensitivity may provide a feasible indication of PARPi response, a few patients who did not respond to platinum reagents benefited from PARPi $[1,13]$. Detection of HRD (especially BRCA1/2 mutations) remains the most convincing predictor of PARPi responsiveness [13, 38]. However, some HR-deficient patients were insensitive, while some HR-proficient patients benefited from PARPi [13, 14]. RAD51 detection is being considered as a promising approach, but its efficiency is restricted by exogenous stresses such as irradiation [13]. C/ EBP $\beta$ directly modulated the HR pathway and targeted four key HR genes. In addition, C/EBP $\beta$ expression predicts platinum sensitivity and HG-SOC prognosis [15]. The positive relationship between $\mathrm{C} / \mathrm{EBP} \beta$ expression and olaparib resistance was successively validated in HR-proficient ovarian cancer cell lines, primary cultures of HG-SOC cancer tissues, and paired samples from HRD-negative patients. Excluding patients 1\# and 2\#, who were identified to have BRCA1 mutation lately, the effects of C/EBP $\beta$ on PARPi resistance were not affected. Thus, C/EBP $\beta$ detection holds great potential in predicting PARPi responsiveness; however, a large preclinical study is required for further confirmation. Furthermore, C/EBP $\beta$ detection using immunohistochemistry might be more cost-effective than gene screening in predicting and tracking PARPi responsiveness during treatment.

C/EBP $\beta$, a potential therapeutic target in epithelial cancers, was reported to control cell proliferation and apoptosis [16]. 
The findings herein indicate that $\mathrm{C} / \mathrm{EBP} \beta$ inhibits apoptosis. Overexpressed $\mathrm{C} / \mathrm{EBP} \beta$ could suppress caspase-dependent induction of apoptosis. Reduced apoptosis in $\mathrm{C} /$ EBP $\beta$-overexpressing cells contributed to $\mathrm{C} / \mathrm{EBP} \beta$-mediated PARPi resistance. However, we could not determine the effect of $\mathrm{C} / \mathrm{EBP} \beta$ on proliferation. The number of clinical specimens and animal models was limited, and these factors could confound the results. Work is ongoing in a large cohort to determine whether $\mathrm{C} / \mathrm{EBP} \beta$ affects cell proliferation in $\mathrm{HG}$ SOC. The underlying mechanisms of C/EBP $\beta$ upregulation upon PARPi treatment were complicated. There was the possibility that, under the selection pressure of PARPi, cells with higher endogenous $\mathrm{C} / \mathrm{EBP} \beta$ expression survived, leading to $\mathrm{C} / \mathrm{EBP} \beta$ upregulation upon PARPi treatment. Besides, the $\mathrm{C} / \mathrm{EBP} \beta$-mediated apoptosis inhibition largely selected cells with overexpressed $\mathrm{C} / \mathrm{EBP} \beta$ for survival, which might also partially explain the phenomenon. $\mathrm{C} / \mathrm{EBP} \beta$ was found to be involved in epigenetic reprogramming [15]. The histone demethylase JMJD3 could escalate C/EBP $\beta$ expression [42]. Insulin and the PI3K/AKT axis were also reported to be implicated in C/EBP $\beta$ upregulation [43]. The potential mechanisms of PARPi-induced $\mathrm{C} / \mathrm{EBP} \beta$ elevation in ovarian cancer remain to be elucidated.

Owing to the significant heterogeneity, using appropriate cell lines to study HG-SOC is challenging [28]. Cell lines are extensively involved in preclinical research. However, we must acknowledge the insufficiency of this study. Based on genomic profiles, A2780 and SKOV3 were reported as unlikely HG-SOC cell lines [44]. A recent study, which integrated genomic, epigenomic, and expression analysis, has proposed that using these well-characterized cell lines during preclinical studies will provide translational rationales for anti-cancer treatment [45]. SKOV3 was revised as a possible HG-SOC cell line since there is a frameshift TP53 mutation [45]. Given the origin of $\mathrm{C}{ }^{*}$ and OV2008, their usage is somewhat indecent [46]. Although the primarily used cell line models had some controversies, the reciprocal relationship between $\mathrm{C} / \mathrm{EBP} \beta$ and PARPi responsiveness was confirmed in several likely/possible HG-SOC cell lines and primary cultures. Deriving cell lines from primary ovarian cancers could minimize the discrepancy between cell line models and clinical tumors [44]. Besides, in the current study, we focused on the HR pathway. However, the relationship between $\mathrm{C} / \mathrm{EBP} \beta$ and replication fork protection and other DDR pathways remains unclear. Although there are difficulties to specifically directly target C/EBP $\beta$ [16], Withaferin A might potentially suppress $\mathrm{C} / \mathrm{EBP} \beta[47,48]$, JMJD3 targets and upregulates C/EBP $\beta$ expression in leukemia [42], and JAK-STAT3 signaling can activate C/EBP $\beta$ [49]. Withaferin A and inhibitors of JMJD3 and STAT3 offer good options for using C/EBP $\beta$ as a therapeutic target. We will be exploring the aforementioned avenues in our future studies on $\mathrm{C} / \mathrm{EBP} \beta$-targeted therapy.
In summary, $\mathrm{C} / \mathrm{EBP} \beta$ is an important regulator of the $\mathrm{HR}$ pathway and promotes PARPi resistance in ovarian cancer. Our research provides evidence that $\mathrm{C} / \mathrm{EBP} \beta$ could be exploited as an indicator of PARPi responsiveness and a therapeutic target. $\mathrm{C} / \mathrm{EBP} \beta$ detection holds great potential in predicting and tracking PARPi responsiveness during treatment, which warrants further investigations. Targeting $\mathrm{C} / \mathrm{EBP} \beta$ could induce HRD and rescue PARPi resistance, which shows promise for extending the benefits of PARPi to a wider population.

\section{Materials and methods}

\section{Clinical samples and primary culture}

All clinical samples were obtained with signed informed consent from the Gynecology Department at Tongji Hospital (Tongji Medical College, Huazhong University of Science and Technology). The study was supervised by the Ethical Committee of Tongji Medical College (reference number: S1251). All the patients included were diagnosed with HG-SOC and had no prior history of chemotherapy or radiotherapy. Six tissue samples (Patient 1\# - 6\#) obtained after fresh frozen pathological examination were used for primary culture as reported previously [50]. The epithelial content reached $80 \%$ after ten times of serial subculture [50], and was confirmed using EPCAM staining. Six paired samples were obtained at initial surgery and subsequent biopsy. These six patients had HR-proficient HG-SOC and had undergone several rounds of olaparib treatment until disease progression. Patients characteristics were summarized in Supplementary Tables 6 and 7.

\section{RNA-sequencing and online database analysis}

RNA-sequencing was performed by BerryGenomics (China). Each sample had eight biological replicates unless otherwise indicated. DEGs were identified as reported by Schurch et al. using the edgeR (exact) algorithm [51]. Detailed information of RNA-sequencing was described in supplementary information.

Cistrome Data Browser (DB) and JASPAR were used to predict the binding affinity and profile of $\mathrm{C} / \mathrm{EBP} \beta[52,53]$. The correlation between C/EBP $\beta$ and DDR genes and their gene expression profiles in TCGA dataset were assessed using GEPIA [54], which is an online database containing RNA-sequencing expression data of 9736 tumor samples and 8587 normal samples. The relationship between C/EBP $\beta$ and olaparib was explored in DepMap (https://depmap.org/ portal/), which provides an overview of dependencies operative in cancer cells for new and effective targeted therapies development. 


\section{Primers and plasmid constructs}

Primers used in Real-time quantitative reverse transcription PCR were listed in Supplementary Table 8. Primers used in ChIP-qPCR were listed in Supplementary Table 9. Detailed information of the promoter regions used in luciferase reporter assay was depicted in Supplementary Table 10.

\section{Animal studies}

Female NOD-SCID mice (age, four weeks) were purchased from Beijing HFK Bio-Technology Co. Ltd (China) and housed at an accredited facility at Tongji Hospital. The animal experiments were approved by the Committee on Ethics of Animal Experiments of Tongji Hospital. Stably transfected cells $\left(1 \times 10^{6}\right)$ were subcutaneously injected into mouse flanks in a mixture of phosphate-buffered saline (PBS) and Matrigel (Corning, USA) [27]. When the tumor size reached $200 \mathrm{~mm}^{3}$, the drug administration schedule reported by Yasukawa et al. was used [55]. The mice were randomly assigned to two groups (six mice per group): (1) olaparib $(30 \mathrm{mg} / \mathrm{kg}$ per body weight, dissolved in $200 \mu \mathrm{l}$ sterilized PBS); (2) vehicle $(200 \mu \mathrm{l}$ sterilized PBS containing an equal concentration of dimethyl sulfoxide [DMSO]). The chemicals were intraperitoneally administered every 2 days for 40 days. The tumors were inspected in living mice by optical imaging of luciferase activity with the IVIS SPECTRUM system (Caliper, Xenogen, USA). The total flux was quantified. After imaging, mice were sacrificed for tissue harvest. Survival analysis was also performed, with survival cut-off criteria defined as tumor volume reaching $1500 \mathrm{~mm}^{3}$ or an observation duration of 90 days.

\section{Statistical analysis}

A two-sided Student's $t$ test was used to compare differences between groups unless otherwise indicated. Correlation analyses were performed using Pearson's correlation test. For the Venn diagram, the chi-squared test was used to evaluate the statistical significance. By Kaplan-Meier plot of survival, Log-rank test was adopted to examine the difference. All experiments were repeated three times unless otherwise indicated. Data were analyzed and plotted using GraphPad Prism 5 (GraphPad Software, San Diego, CA) and presented as mean \pm SEM. Significance was assessed at the level of $P<0.05$.

\section{Data availability}

The RNA-sequencing dataset was deposited to GEO with accession number GSE153867.
Acknowledgements This work was supported by National Sci-Tech Support Projects (2018ZX10301402-002), National Key Basic Research Program of China (2015CB553903), National Natural Science Foundation of China (82072889, 81974405, and 81772787), Chinese Society of Clinical Oncology (Y-2019AZZD-0359), Leading Talents of Science and Technology Innovation in National Ten Thousand Talents Plan ([2019]252), and Tongji Hospital (HUST) Foundation for Excellent Young Scientist (2020YBKY024). We sincerely thank all participants in the study.

Author contributions This study was designed by DM, DL, QG, and JT. JT, XZ, ML, and CS did the experiments. JT, XZ, FY, CX, XZ, WL, YW, SZ, HL, GC, XH, DL, and QG analyzed and interpreted the data. DL and QG conceptualized and supervised the project. JT, XZ, $\mathrm{DL}$, and QG were major contributors in writing the manuscript. The corresponding authors have full access to the data in the study and final responsibility for the decision to submit for publication.

\section{Compliance with ethical standards}

Conflict of interest The authors declare no competing interests.

Publisher's note Springer Nature remains neutral with regard to jurisdictional claims in published maps and institutional affiliations.

Open Access This article is licensed under a Creative Commons Attribution 4.0 International License, which permits use, sharing, adaptation, distribution and reproduction in any medium or format, as long as you give appropriate credit to the original author(s) and the source, provide a link to the Creative Commons license, and indicate if changes were made. The images or other third party material in this article are included in the article's Creative Commons license, unless indicated otherwise in a credit line to the material. If material is not included in the article's Creative Commons license and your intended use is not permitted by statutory regulation or exceeds the permitted use, you will need to obtain permission directly from the copyright holder. To view a copy of this license, visit http://creativecommons. org/licenses/by/4.0/.

\section{References}

1. Lheureux S, Braunstein M, Oza AM. Epithelial ovarian cancer: evolution of management in the era of precision medicine. CA Cancer J Clin. 2019;69:280-304.

2. Lheureux S, Gourley C, Vergote I, Oza AM. Epithelial ovarian cancer. Lancet. 2019;393:1240-53.

3. Bian L, Meng Y, Zhang M, Li D. MRE11-RAD50-NBS1 complex alterations and DNA damage response: implications for cancer treatment. Mol Cancer. 2019;18:169.

4. Curtin NJ. DNA repair dysregulation from cancer driver to therapeutic target. Nat Rev Cancer. 2012;12:801-17.

5. Li A, Yi M, Qin S, Chu Q, Luo S, Wu K. Prospects for combining immune checkpoint blockade with PARP inhibition. J Hematol Oncol. 2019;12:98.

6. Lord CJ, Ashworth A. PARP inhibitors: synthetic lethality in the clinic. Science. 2017;355:1152-8.

7. McCabe N, Turner NC, Lord CJ, Kluzek K, Bialkowska A, Swift $\mathrm{S}$, et al. Deficiency in the repair of DNA damage by homologous recombination and sensitivity to poly(ADP-ribose) polymerase inhibition. Cancer Res. 2006;66:8109-15.

8. Cancer Genome Atlas Research N. Integrated genomic analyses of ovarian carcinoma. Nature. 2011;474:609-15. 
9. Yuan Y, Liao Y-M, Hsueh C-T, Mirshahidi HR. Novel targeted therapeutics: inhibitors of MDM2, ALK and PARP. J Hematol Oncol. 2011;4:16.

10. Cortez AJ, Tudrej P, Kujawa KA, Lisowska KM. Advances in ovarian cancer therapy. Cancer Chemother Pharm. 2018; $81: 17-38$

11. Lord CJ, Ashworth A. BRCAness revisited. Nat Rev Cancer. 2016;16:110-20.

12. Konecny GE. Combining PARP and CDK4/6 inhibitors in MYC driven ovarian cancer. EBioMedicine. 2019;43:9-10.

13. Sun C, Cao W, Qiu C, Li C, Dongol S, Zhang Z, et al. MiR-509-3 augments the synthetic lethality of PARPi by regulating HR repair in PDX model of HGSOC. J Hematol Oncol. 2020;13:9.

14. Zhu H, Wei M, Xu J, Hua J, Liang C, Meng Q, et al. PARP inhibitors in pancreatic cancer: molecular mechanisms and clinical applications. Mol Cancer. 2020;19:49.

15. Liu D, Zhang XX, Li MC, Cao CH, Wan DY, Xi BX, et al. C/ EBPbeta enhances platinum resistance of ovarian cancer cells by reprogramming H3K79 methylation. Nat Commun. 2018;9:1739.

16. Zahnow CA. CCAAT/enhancer-binding protein beta: its role in breast cancer and associations with receptor tyrosine kinases. Expert Rev Mol Med. 2009;11:e12.

17. Ji T, Gong D, Han Z, Wei X, Yan Y, Ye F, et al. Abrogation of constitutive Stat 3 activity circumvents cisplatin resistant ovarian cancer. Cancer Lett. 2013;341:231-9.

18. Pignochino Y, Capozzi F, D'Ambrosio L, Dell'Aglio C, Basirico $\mathrm{M}$, Canta $\mathrm{M}$, et al. PARP1 expression drives the synergistic antitumor activity of trabectedin and PARP1 inhibitors in sarcoma preclinical models. Mol Cancer. 2017;16:86.

19. He X, Zhang P. Serine/arginine-rich splicing factor 3 (SRSF3) regulates homologous recombination-mediated DNA repair. Mol Cancer. 2015;14:158.

20. Lord CJ, Ashworth A. The DNA damage response and cancer therapy. Nature. 2012;481:287-94.

21. Ceccaldi R, Rondinelli B, D'Andrea AD. Repair pathway choices and consequences at the double-strand break. Trends Cell Biol. 2016;26:52-64.

22. Taverna P, Liu L, Hanson AJ, Monks A, Gerson SL. Characterization of MLH1 and MSH2 DNA mismatch repair proteins in cell lines of the NCI anticancer drug screen. Cancer Chemother Pharm. 2000;46:507-16.

23. Peasland A, Wang LZ, Rowling E, Kyle S, Chen T, Hopkins A, et al. Identification and evaluation of a potent novel ATR inhibitor, NU6027, in breast and ovarian cancer cell lines. $\mathrm{Br} \mathrm{J}$ Cancer. 2011;105:372-81.

24. Aebi S, Kurdi-Haidar B, Gordon R, Cenni B, Zheng H, Fink D, et al. Loss of DNA mismatch repair in acquired resistance to cisplatin. Cancer Res. 1996;56:3087-90.

25. Yamaguchi H, Du Y, Nakai K, Ding M, Chang SS, Hsu JL, et al. EZH2 contributes to the response to PARP inhibitors through its PARP-mediated poly-ADP ribosylation in breast cancer. Oncogene. 2018;37:208-17.

26. Kim H, George E, Ragland R, Rafail S, Zhang R, Krepler C, et al. Targeting the ATR/CHK1 axis with PARP inhibition results in tumor regression in BRCA-mutant ovarian cancer models. Clin Cancer Res: Off J Am Assoc Cancer Res. 2017;23:3097-108.

27. Sun C, Yin J, Fang Y, Chen J, Jeong KJ, Chen X, et al. BRD4 inhibition is synthetic lethal with PARP inhibitors through the induction of homologous recombination deficiency. Cancer Cell. 2018;33:401-416 e408.

28. Yi J, Liu C, Tao Z, Wang M, Jia Y, Sang X, et al. MYC status as a determinant of synergistic response to Olaparib and Palbociclib in ovarian cancer. EBioMedicine. 2019;43:225-37.

29. Dong Q, Du Y, Li H, Liu C, Wei Y, Chen MK, et al. EGFR and cMET cooperate to enhance resistance to PARP inhibitors in hepatocellular carcinoma. Cancer Res. 2019;79:819-29.
30. Marques M, Jangal M, Wang LC, Kazanets A, da Silva SD, Zhao $\mathrm{T}$, et al. Oncogenic activity of poly (ADP-ribose) glycohydrolase. Oncogene. 2019;38:2177-91.

31. Fang Y, McGrail DJ, Sun C, Labrie M, Chen X, Zhang D, et al. Sequential therapy with PARP and WEE1 inhibitors minimizes toxicity while maintaining efficacy. Cancer Cell. 2019; 35:851-867 e857.

32. Dhawan MS, Bartelink IH, Aggarwal RR, Leng J, Zhang JZ, Pawlowska N, et al. Differential toxicity in patients with and without DNA repair mutations: phase i study of carboplatin and talazoparib in advanced solid tumors. Clin Cancer Res. 2017;23:6400-10.

33. Miller AL, Fehling SC, Garcia PL, Gamblin TL, Council LN, van Waardenburg RCAM, et al. The BET inhibitor JQ1 attenuates double-strand break repair and sensitizes models of pancreatic ductal adenocarcinoma to PARP inhibitors. EBioMedicine. 2019;44:419-30.

34. Stewart RA, Pilie PG, Yap TA. Development of PARP and immune-checkpoint inhibitor combinations. Cancer Res. 2018;78:6717-25.

35. Bian X, Gao J, Luo F, Rui C, Zheng T, Wang D, et al. PTEN deficiency sensitizes endometrioid endometrial cancer to compound PARP-PI3K inhibition but not PARP inhibition as monotherapy. Oncogene. 2018;37:341-51.

36. Penning TD. Small-molecule PARP modulators-current status and future therapeutic potential. Curr Opin drug Discov Dev. 2010;13:577-86.

37. Shen Y, Aoyagi-Scharber M, Wang B. Trapping poly(ADPRibose) polymerase. J Pharm Exp Ther. 2015;353:446-57.

38. Robinson LS, Hendrix A, Xie X-J, Yan J, Pirzadeh-Miller S, Pritzlaff $\mathrm{M}$, et al. Prediction of cancer prevention: from mammogram screening to identification of BRCA1/2 mutation carriers in underserved populations. EBioMedicine. 2015;2:1827-33.

39. Murai J, Huang SY, Das BB, Renaud A, Zhang Y, Doroshow JH, et al. Trapping of PARP1 and PARP2 by clinical PARP inhibitors. Cancer Res. 2012;72:5588-99.

40. Liu Q, Gheorghiu L, Drumm M, Clayman R, Eidelman A, Wszolek MF, et al. PARP-1 inhibition with or without ionizing radiation confers reactive oxygen species-mediated cytotoxicity preferentially to cancer cells with mutant TP53. Oncogene. 2018;37:2793-805.

41. Wang J, Kho DH, Zhou JY, Davis RJ, Wu GS. MKP-1 suppresses PARP-1 degradation to mediate cisplatin resistance. Oncogene. 2017;36:5939-47.

42. Yu SH, Zhu KY, Chen J, Liu XZ, Xu PF, Zhang W, et al. JMJD3 facilitates $\mathrm{C} / \mathrm{EBPbeta}$-centered transcriptional program to exert oncorepressor activity in AML. Nat Commun. 2018;9:3369.

43. Sato Y, Nishio Y, Sekine O, Kodama K, Nagai Y, Nakamura T, et al. Increased expression of CCAAT/enhancer binding proteinbeta and -delta and monocyte chemoattractant protein- 1 genes in aortas from hyperinsulinaemic rats. Diabetologia. 2007;50:481-9.

44. Domcke S, Sinha R, Levine DA, Sander C, Schultz N. Evaluating cell lines as tumour models by comparison of genomic profiles. Nat Commun. 2013;4:2126.

45. Papp E, Hallberg D, Konecny GE, Bruhm DC, Adleff V, Noe M, et al. Integrated genomic, epigenomic, and expression analyses of ovarian cancer cell lines. Cell Rep. 2018;25:2617-33.

46. Korch C, Spillman MA, Jackson TA, Jacobsen BM, Murphy SK, Lessey BA, et al. DNA profiling analysis of endometrial and ovarian cell lines reveals misidentification, redundancy and contamination. Gynecol Oncol. 2012;127:241-8.

47. Rosen ED, Hsu CH, Wang X, Sakai S, Freeman MW, Gonzalez FJ, et al. C/EBPalpha induces adipogenesis through PPARgamma: a unified pathway. Genes Dev. 2002;16:22-26.

48. Lee IC, Choi BY. Withaferin-A-A natural anticancer agent with pleitropic mechanisms of action. Int J Mol Sci. 2016;17:290. 
49. Wang ZH, Xiang J, Liu X, Yu SP, Manfredsson FP, Sandoval IM, et al. Deficiency in BDNF/TrkB neurotrophic activity stimulates delta-secretase by upregulating C/EBPbeta in Alzheimer's disease. Cell Rep. 2019;28:655-669 e655.

50. Kurbacher CM, Korn C, Dexel S, Schween U, Kurbacher JA, Reichelt R, et al. Isolation and culture of ovarian cancer cells and cell lines. Methods Mol Biol. 2011;731:161-80.

51. Schurch NJ, Schofield P, Gierlinski M, Cole C, Sherstnev A, Singh V, et al. How many biological replicates are needed in an RNA-seq experiment and which differential expression tool should you use? RNA. 2016;22:839-51.

52. Zheng R, Wan C, Mei S, Qin Q, Wu Q, Sun H, et al. Cistrome data browser: expanded datasets and new tools for gene regulatory analysis. Nucleic Acids Res. 2019;47: D729-D735.

53. Fornes O, Castro-Mondragon JA, Khan A, van der Lee R, Zhang X, Richmond PA, et al. JASPAR 2020: update of the open-access database of transcription factor binding profiles. Nucleic Acids Res. 2020;48:D87-D92.

54. Tang Z, Li C, Kang B, Gao G, Li C, Zhang Z. GEPIA: a web server for cancer and normal gene expression profiling and interactive analyses. Nucleic Acids Res. 2017;45:W98-W102.

55. Yasukawa M, Fujihara H, Fujimori H, Kawaguchi K, Yamada H, Nakayama R, et al. Synergetic effects of PARP inhibitor AZD2281 and cisplatin in oral squamous cell carcinoma in vitro and in vivo. Int J Mol Sci. 2016;17:272. 